\title{
Electropulsing-induced Phase Transformation in Titanium Alloy
}

\author{
W. Zhang, M.L. Sui, W.S. Zhao and D.X. Li
}

Shenyang National Laboratory for Materials Science, Institute of Metal Research, Chinese Academy of Sciences, 72 Wenhua Road, Shenyang 110016, China.

Solid-state phase transformations under the electropulsing have been extensively researched [1-2]. However, our understanding of the detailed atomic mechanisms pertaining to the effects of a current on phase transformation is still very rudimentary hitherto. Especial needs are careful and definitive high-resolution transmission electron microscopy (HRTEM) observations of the transformation products under the electropulsing [1]. In this paper, by means of HRTEM an investigation of phase transformation in Ti-6Al-4V alloy induced by the electropulsing was reported.

The electropulsing was performed at ambient conditions, by the capacitor $(1200 \mu \mathrm{F})$ discharging with Xe flasher trigger. A commercial Ti-6Al-4V alloy plate with a thickness of $1 \mathrm{~mm}$ was used in this work.

The original sample consists of equal-axial $\alpha$-Ti phase and a small amount of intergranular $\beta$-Ti phase, as shown in Fig 1. However, many ultra-fine $\beta$-Ti laths occurred in the interior of $\alpha$ grains under electropulsing, revealing an obvious phase transformation from $\alpha$-Ti to $\beta$-Ti as shown in Fig 2(a). From the diffraction pattern shown in Fig 2(b), it was found that the orientation relationship (OR) between $\alpha$-Ti and $\beta$-Ti phases is that $(1100)_{\alpha-\mathrm{Ti}} / /(112)_{\beta \text {-Ti }},[0001]_{\alpha-\mathrm{Ti}} / /[110]_{\beta-\mathrm{Ti}}$, instead of the conventional Burgers relation $(0001)_{\alpha-\mathrm{Ti}} /(110)_{\beta-\mathrm{Ti}},[11 \overline{2} 0]_{\alpha-\mathrm{Ti}} / /[\overline{11} 1]_{\beta-\mathrm{Ti}}$ in titanium alloy [3], as shown in Fig 3(a). These can result from the Joule heat, instantaneous thermal stress and some other effects from the electropulsing. It was also found that there exists the $(\overline{11})_{\beta \text {-Ti }}$ twinning between many adjacent $\beta$-Ti laths, in which the dashed lines represent (112) twinning planes as shown in Fig 3 (b). It is known that it is difficult to twin in b.c.c. $\beta$-Ti phase, and therefore large amounts of $\beta$-Ti twins may be introduced from the considerable stress concentration under the electropulsing.

In summary, the phase transformation from $\alpha$-Ti to $\beta$-Ti has occurred in the Ti-6Al-4V alloy under the electropulsing. The new OR between $\alpha$-Ti and $\beta$-Ti phases is $(1100)_{\alpha-\mathrm{Ti}} /(\overline{11} 12)_{\beta-\mathrm{Ti}}$, $[0001]_{\alpha-\mathrm{Ti}} / /[110]_{\beta-\mathrm{Ti}}$. Also there exists the $\{112\}$ twinning in the formed $\beta$-Ti phase [4].

\section{References}

[1] H. Conrad, Mater. Sci. Eng. A287 (2000) 227.

[2] R. Takemoto, and H. Mizubayashi, Acta. Metall. Mater. 43 (1995) 1495.

[3] W.G. Burgers, Physica 1 (1934) 561.

[4] This research was supported by NSFC (90206044, 50271074 and 50125103). 


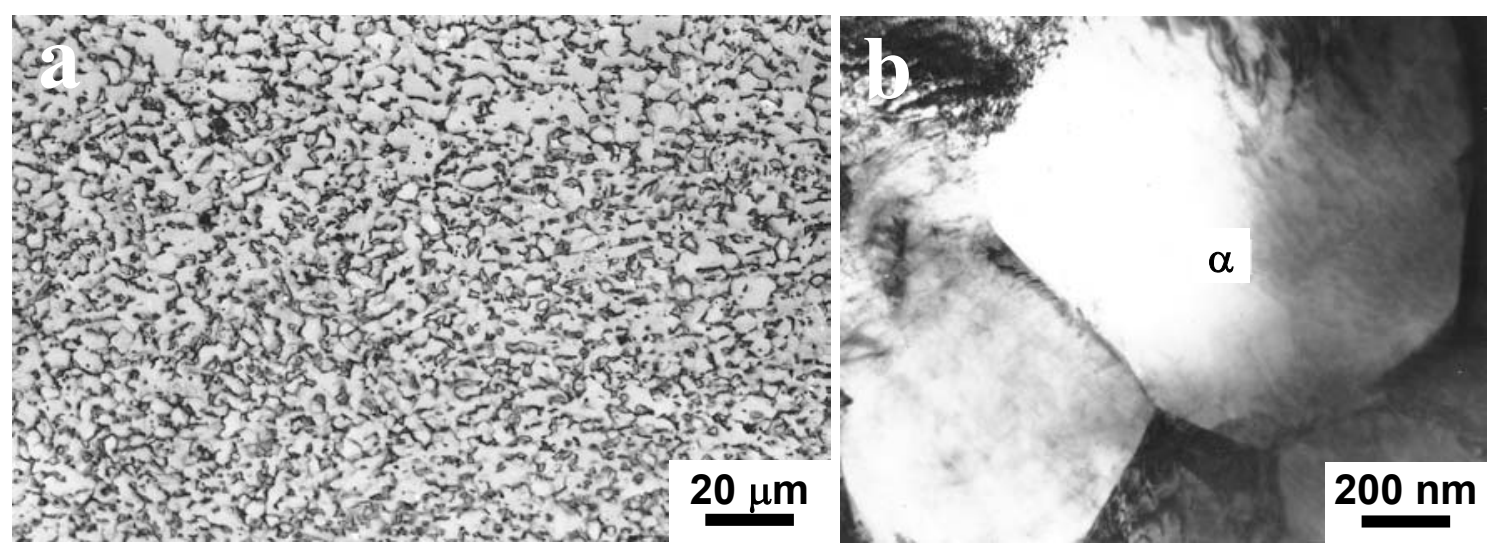

FIG. 1. Light micrograph (a) and typical TEM image (b) of the alloy before the electropulsing.


FIG. 2. TEM image (a) and corresponding electron diffraction pattern (b) of the basket-weave microstructure in the alloy after the electropulsing.


FIG. 3. HREM images of the new OR between $\alpha$-Ti and $\beta$-Ti phases (a) and $\beta$-Ti twins (b) in the microstructure of Fig 2(a). 